\title{
Stratified Treatment in Lung Cancer
}

\author{
Sebastian Michels Jürgen Wolf \\ Lung Cancer Group Cologne, Department I for Internal Medicine, Center for Integrated Oncology, University Hospital of Cologne, \\ Cologne, Germany
}

Keywords

Lung cancer, stratified therapy

\section{Summary}

Even though great efforts have been made to improve chemotherapy-based treatment approaches for lung cancer, the prognosis of patients with advanced and metastasized disease remains particularly poor. In recent years, a growing number of genetic aberrations driving lung cancer have been identified. Targeted inhibition of some of these aberrations, most prominently mutated EGFR and ALK, by tyrosine kinase inhibitors has dramatically increased efficacy and tolerability of systemic lung cancer treatment in subsets of patients. However, the duration of response is limited due to the acquisition of molecular mechanisms of resistance to targeted treatment. Modern next-generation inhibitors aim to break resistance. A deep understanding of the mechanisms of treatment failure is imperative to the development of new approaches. In this review, we focus on the current status of stratified therapy in lung cancer and highlight new, potentially promising treatment approaches.

(c) 2016 S. Karger GmbH, Freiburg

\section{Principles of Stratified Medicine}

While for decades lung cancer subtype classification and therapeutic strategies were restricted to histology and morphology only, the disease now has turned out to be highly heterogeneous on the genomic level. This development does not only help us to understand the clinical heterogeneity of lung cancer, but also enables us to achieve therapeutic breakthrough advances by developing biological-rational treatment approaches based on a precise understanding of the molecular mechanisms underlying malignant transformation and its inhibition.

\section{KARGER}

(c) 2016 S. Karger GmbH, Freiburg
First-line treatment with cisplatin and etoposide has been standard of care for metastasized small cell (SCLC) and non-small cell lung cancer (NSCLC) for many years. This paradigm changed with the introduction of second-generation chemotherapeutic agents such as paclitaxel, docetaxel, and gemcitabine. Platinumcombinations with these drugs were shown to be superior to cisplatin and etoposide in NSCLC patients and defined new standards [1-4]. Newer chemotherapeutic agents, such as pemetrexed and antiangiogenic drugs, have moderately increased efficacy and tolerability of conventional treatment predominantly in adenocarcinoma [5-9]. However, response rates and overall survival have only marginally improved with non-stratified treatment approaches. With a range of 1 year, median overall survival remains almost unchanged and it becomes evident that the limits of conventional systemic approaches have been reached.

Since the discovery of oncogenic EGFR mutations in a distinct subgroup of NSCLC patients and the first evidence of the high efficacy of EGFR inhibition in these, a big step forward has been made for an increasing number of genetically defined NSCLC sub-entities. More and more molecularly stratified treatment strategies were successfully tested and implemented into clinical routine in the recent years [10-18]. Broad comprehensive analyses of patients' characteristics and genetic tumor profiles were undertaken by several groups in order to better understand the natural history of lung cancer and the characteristics of the different genetic subgroups [19-21]. Large molecular screening and treatment networks have been established in different countries to implement comprehensive genetic testing and stratified treatment into clinical routine. Through these efforts the number of patients receiving stratified treatment increased steadily to about $20 \%$ of all lung cancer patients nowadays. However, for the majority of patients no promising targeted treatment approaches could be established so far, including tumors with complex genetic profiles like aberrations in tumor suppressor genes or G-proteins, which are mostly found in tobacco-triggered disease [22]. For these patients, immune checkpoint inhibitors are now a new and promising option. This review will focus on stratified therapies directed against transforming genetic aberrations. 
Stratified treatment follows a recurrent principle, which should not be confounded with targeted treatment in unselected patients, e.g. antiangiogenic treatment. The difference lies in the identification of molecular targets triggering oncogenic growth (dependency) and thus predicting efficacy through specific inhibition (vulnerability).

\section{Resistance and New Generation TKls}

Like other malignancies, lung cancer is characterized by a high genomic instability and increased mutation rate [23]. The constant acquisition of genetic aberrations and treatment pressure by targeted antineoplastic drugs result in the selection of desensitized tumor cells following Darwinian rules [24]. Following stratified tyrosine kinase inhibitor (TKI) treatment, resistance is often mediated through secondary mutations in the target gene preventing the enzymatic inhibition [25]. Substitution of the 'gatekeeper' threonine for example is a recurrent pattern in different cancer entities after treatment with targeted drugs [25].

Next-generation TKIs are designed to overcome resistance mediated by secondary mutations and have shown to be highly successful in NSCLC [25-28].

Owed to intra-tumor heterogeneity, resistance may alternatively be mediated by aberrations independent of the target gene or by more than one mechanism simultaneously $[29,30]$. This complexity represents a growing challenge and combination therapies are tested to overcome resistance through multiple factors [29, 31, 32]. The heterogeneous nature of resistance makes tissue analysis by thorough genetic profiling indispensable to treatment decision-making.

\section{EGFR}

Mutations in EGFR have been the first targetable aberrations to be identified in lung cancer $[33,34]$. Activating EGFR mutations are detected in approximately $10 \%$ of Caucasian NSCLC patients and the most common aberrations include the substitution L858R in exon 21 and a variety of small deletions in exon 19 [19-21, 33, 34]. With the majority of EGFR-mutant NSCLC patients being non-smokers and female, this subgroup is characterized by distinct clinico-pathological features [19-21, 35]. Superiority in terms of response rates and progression-free survival (PFS) of the first-generation TKIs gefitinib and erlotinib in first-line compared to chemotherapy was proven in several trials $[10-13,15,36]$. However, due to cross-over of chemotherapy patients to TKI therapy after progression, overall survival (OS) was not significantly improved with either gefitinib or erlotinib. In a cohort analysis of the LUX-Lung 3 and 6 trials, the second-generation inhibitor afatinib was shown to prolong OS in EGFR del19-positive NSCLC patients [37]. Although an OS benefit could not be demonstrated in randomized trials, there is no doubt that treatment with any EGFR TKI significantly prolongs OS in EGFR-mutated patients compared to chemotherapy. In a retrospective analysis of the German Network Genomic Medicine, the median OS with chemotherapy treatment was significantly longer with EGFR TKI treat- ment as compared to chemotherapy (31.5 vs. 9.6 months, HR 0.169 ) [21]. A comparable OS benefit was shown in analyses by the US Lung Cancer Mutational Consortium and the French Cooperative Thoracic Intergroup [19, 20].

Even though big advances were made with the clinical use of EGFR TKIs, median PFS does not exceed 12-14 months and treatment inevitably fails due to the acquisition of molecular resistance. In approximately $60 \%$ of cases a secondary mutation of the 'gatekeeper' threonine in exon 20 of EGFR, EGFR T790M, is responsible for the desensitization to EGFR TKIs [25, 38, 39]. Modern thirdgeneration TKIs are able to overcome resistance in patients with EGFR T790M-positive NSCLC [27, 28]. Osimertinib, so far the only drug approved in this setting by the Federal Drug and Food Administration (FDA) and the European Medicines Agency (EMA), showed a response rate (RR) of $61 \%$ and a PFS of 9.6 months in a phase I trial [40]. Other third-generation EGFR TKIs such as EGF816 and olmutinib (BI 1482694/HM61713) are in clinical development and show similar results regarding efficacy and safety [41-43].

Resistance to EGFR TKI may not be restricted to the acquisition of a single mechanism. Co-occurrance of EGFR T790M and other mechanisms such as amplification of MET and HER2 or the activation of the MEK/ERK pathway were found in subsets of patients [29-32, 44]. Single agent treatment with third-generation EGFR TKIs seems to be insufficient in this setting [29, 30, 32, $44,45]$. Trials combining TKIs that target EGFR as well as MET or MEK are currently enrolling patients (NCT02143466; NCT02335944).

Several mechanisms of acquired resistance to third-generation TKIs were recently identified, including the same EGFR-independent mechanisms that mediate resistance to first- or second-generation EGFR TKIs [29, 44]. The substitution mutation EGFR C797S inhibits third-generation EGFR TKIs to bind to the protein and block the enzymatic activity, thus mediating resistance to the treatment $[46,47]$. Currently, no drug is in clinical testing to break this mechanism of acquired resistance.

Whether third-generation EGFR-TKIs will be used in first-line, is a topic of ongoing discussion. Preliminary results of osimertinib in first-line treatment, showing a PFS of about 20 months are promising [48].

\section{$A L K$}

Rearrangements of the $A L K$ gene predominantly affect neversmokers of younger age and are found in approximately $5 \%$ of NSCLC patients [19-21, 49-51]. Upon the results of superiority in efficacy (RR, $74 \%$ vs. $45 \%$ and PFS, 10.9 vs. 7.0 months; HR, 0.45) and safety of first-line crizotinib compared to platinum-based chemotherapy, the drug recently received approval by the EMA and the FDA for expanded use in ALK-positive patients [16, 52].

Acquisition of secondary mutations in $A L K$ is a common mechanism of resistance to crizotinib $[26,53,54]$. However, resistance factors independent of $A L K$ have been described [53]. The development and approval of next-generation ALK inhibitors, that break resistance to crizotinib, has been remarkably fast. With re- 
sponse rates around 50\% the second-generation TKIs ceritinib and alectinib, have shown high efficacy in the setting of crizotinib resistance $[17,18,55]$.

Up to $30 \%$ of patients with ALK-rearranged NSCLC have brain metastases at baseline and a large part of patients treated with crizotinib will experience isolated progression of central nervous system (CNS) metastases [56-58]. Intracranial RR of crizotinib in patients with untreated CNS metastases is $18 \%$ and thus much lower than the extracranial RR [57]. A possible explanation for the recurrent CNS failure of crizotinib may lie in the low blood-brain-barrier penetration of the drug $[56,57]$. Second-generation ALK inhibitors exhibit a higher CNS penetration and subgroup analyses of study data suggest a higher activity in brain metastases than crizotinib $[58,59]$. Still, the question will need further clinical investigation and low patient numbers as well as heterogeneous patient selection make comparison between the different trials difficult.

At this time, 2 potential treatment alternatives remain in patients with isolated CNS progression - treatment beyond progression with crizotinib and local treatment of CNS metastases or switch to a CNS penetrable ALK TKI [55]. Recently, the next-generation ALK inhibitors lorlatinib and brigatinib have been brought into clinical evaluation (NCT01970865; NCT01449461; NCT02737501). First results of lorlatinib showed RR of $42 \%$ in patients who exhibit progression on 2 or more ALK TKIs. In patients who have received treatment with $1 \mathrm{TKI}$, lorlatinib and brigatinib show similar RR around $60 \%[58,60]$.

\section{ROS1}

Like aberrations in EGFR and $A L K$ oncogenic rearrangements of ROS1 predominantly affect young never-smokers and are associated with a favorable prognosis. Prevalence is low, ranging between 1 and $2 \%$ of the overall NSCLC population $[45,49,61,62]$. The ALK/ROS1/MET inhibitor crizotinib is the first drug to show efficacy in this patient subgroup. The phase I trial by Shaw and colleagues found a RR of $72 \%$ with a median PFS of 19.2 months and retrospective analyses showed similar efficacy [63]. Further prospective trials, including an international European trial (EUCROSS; NCT02183870), are testing crizotinib in this patient subgroup and are likely to confirm this data. Recently, the EMA and the FDA granted conditional approval for crizotinib treatment in ROS1 rearranged NSCLC, setting a new standard of care for these patients.

Following the same biological rules, targeted therapy in ROS1positive patients results in the acquisition of resistance [64-68]. Unlike EGFR and ALK TKI resistance, resistance to crizotinib in ROS1-positive patients is not well understood. Only a few secondary mutations in ROS1 have been described so far and ROS1-independent mechanisms of resistance may be an important factor [69, 70]. The multi-target inhibitor cabozantinib showed efficacy in pre-clinical models and single patient cases with acquired resistance to crizotinib and the resistance mutations G2032R, D2033N and L2026M $[65,67,68]$. More selective drugs such as the nextgeneration ALK/ROS1 TKI lorlatinib are in clinical investigation and showed promising efficacy in this setting [71].

\section{MET}

The oncogenic potential of the receptor tyrosine kinase (RTK) MET may be triggered by different mechanisms of activation in multiple tumor entities [72-75]. In NSCLC, activation of MET was reported by overexpression in $22-25 \%$, by high-level amplification in $3 \%$ and most recently by exon 14 skipping mutations in approximately $3 \%$ of patients $[73,76,77]$. Amplification of MET is associated with worse survival after resection and in patients with advanced cancer stage $[73,78]$.

Various MET inhibitors or monoclonal antibodies have been in clinical development for many years now and most have failed to prove convincing efficacy. The most prominent example is the MET antibody onartuzumab [79]. Based on the promising phase II results of onartuzumab in combination with erlotinib in patients with high MET expression, a phase III trial was launched to test this combination in this patient subgroup. The trial was stopped for futility, and a debate emerged on the possible reasons, focusing on the misleading phase II results and the insufficient molecular selection of patients due to unsuccessful definition of biomarkers.

Modern, more potent MET inhibitors such as crizotinib and capmatinib have shown high efficacy in patients with high-level amplification or expression of MET as well as MET splice site mutations in clinical trials, retrospective analyses, and case reports [77, 80-83]. However, further comprehensive analyses are needed to define reliable cut-offs for true $M E T$ positivity predicting response to MET inhibition.

\section{BRAF}

Mutations in BRAF may have different effects on the encoded RTK. Mutations involving codon V600 are activating, whereas mutations involving other bases may be inactivating. However, both kinds of mutations harbor oncogenic potential and were described in $2-4 \%$ of NSCLCs $[19-21,84-86]$. BRAF mutations are predominantly found in adenocarcinomas and patients share similar epidemiological characteristics with the overall NSCLC population. Inhibitors of BRAF have long been used in patients with BRAF V600mutated melanoma $[87,88]$. Recently, clinical trials have shown moderate activity of the BRAF inhibitors dabrafenib and vemurafenib in V600-mutated NSCLC as monotherapy [89, 90]. RR are considerably increased to $63 \%$ when dabrafenib is combined with the MEK inhibitor trametinib, whereas, toxicity profiles are similar. In patients with $B R A F$ V600E-positive lung cancer suffering from relapse after standard chemotherapy, treatment with this combination should be considered either in clinical trials or off-label.

\section{New Targets in SCLC and NSCLC}

The number of biomarkers accessible to targeted treatments is continuously increasing in adenocarcinomas of the lungs. In squamous cell lung cancer and SCLC major screening efforts have long failed to identify targetable aberrations. 
Most recently, a promising targeted approach was brought to clinical development in SCLC. A major fraction of $72-85 \%$ of SCLC shows an overexpression of the delta-like 3 surface protein (DLL3), a marker for neuroendocrine phenotype [91]. These findings led to the development of the antibody-drug conjugate (ADC) rovalpituzumab teserine (Rova-T) targeting DLL3. An early clinical trial presented at ASCO 2016 showed a RR of 30\% in patients with SCLC harboring DLL3 expression of $\geq 50 \%$ independent of the number of prior lines of therapy [92]. Trials further investigating the drug in DLL3-positive SCLC patients in different lines of therapy have been launched (NCT02674568, NCT02819999).

The discovery of amplifications of FGFR1 in SCLC has led to the investigation of several FGFR inhibitors in these tumors [9395]. However, the results of early clinical trials with lucitinib, BGJ398, dovitinib or ADZ4547 in FGFR1-amplified NSCLC have been disappointing [96-98]. Nevertheless, long-lasting responses are proof-of-principle for the activity in patients with FGFR1-amplified NSCLC [98]. Yet, it is unclear what co-occurring factors influence response to FGFR inhibition or whether amplification assessed by FISH is the right biomarker for prediction of response.

Rearrangements of NTRK1, encoding the RTK TRKA have recently been found in $0.1 \%$ of all NSCLC patients and in $3 \%$ of driver-oncogene negative adenocarcinoma patients [99-101]. Patients with NTRK1-rearranged NSCLC are particularly young and predominantly never-smokers. The pan-TRK inhibitors entrectinib and LOXO-101 are subject to clinical development and show promising activity in pre-clinical models and early clinical setting [68, 99, 102-104].

Other genetic aberrations such as mutations in HER2 or rearrangements of RET have been reported in subsets of patients with NSCLC. TKIs and monoclonal antibodies that potentially inhibit the two proteins do exist, but clinical evaluation is still ongoing [49, 105-107].

\section{How and What to Test?}

One of the most crucial challenges of precision medicine lies in the detection of the increasing number of genetic aberrations that are subject to targeted drugs. Currently, conventional approaches by sequential single gene testing are widely applied. Tissue from biopsies of lung cancer is often restricted and requires reasonable processing. The amount of DNA needed for single gene based molecular pathologic characterization of a tumor increases with every sequential analysis. Starting with the most likely aberration, it may be possible that rare oncogenic events are not identified for the lack of further tumor tissue. The main hurdle of modern diagnostics are therefore the need to test an increasing number of genetic aberrations in a limited amount of tumor tissue.

Next generation sequencing (NGS) approaches are able to overcome these difficulties. Targeted massively parallel (MPS) or hybrid-capture based DNA-sequencing allow the analysis of multiple amplicons of a pre-defined subset of relevant genes of a large number of individuals in a single run [19-21, 108, 109]. Aberrations can be detected on single-nucleotide level, such as point mutations in EGFR or KRAS, or at a larger scale, including deletions, amplifications, or rearrangements. In clinical routine, however, amplifications and rearrangements are often detected by fluorescence in-situ hybridization, which is the most evaluated method and still the gold-standard in most cases.

Modern approaches to detect and sequence cell-free tumor DNA (cfDNA) have been developed to detect genetic aberrations on nucleotide level $[110,111]$. The third-generation EGFR inhibitor osimertinib has been approved for the treatment of patients with EGFR T790M detected by cfDNA analyses. However, tumor heterogeneity responsible for more complex resistance profiles, such as MET or HER2 amplification, may not be detected by conventional single gene sequencing technologies [44]. The value of liquid biopsies in the treatment of lung cancer, especially in the resistance setting needs further evaluation.

Recommendations on what genes to test in metastasized NSCLC are inconsistent and underlie constant change. A recently published consensus report from the Molecular Analysis for Personalised Therapy Conference (MAP) recommends testing for mutations in EGFR, ALK, and ROS1 in daily routine but also recommends testing of further 20 genes within screening programs to allow patients the participation in clinical trials or off-label treatment [112]. With an increasing number of potential treatment options as outlined above, determination of EGFR, ALK, and ROS1 status is insufficient nowadays. Large European screening platforms like the Network Genomic Medicine or the French Cooperative Thoracic Intergroup have proven to efficiently perform screening for a large subset of relevant genes in huge numbers of NSCLC patients [19, 21].

\section{Conclusions}

An increased understanding of the molecular mechanisms underlying malignancy has led to a new perception of lung cancer as a disease of many genetically defined subgroups. This has enabled the development of biologically rational stratified treatment strategies against molecular alterations defining these subgroups. Today, about $20 \%$ of lung cancer patients benefit from such approaches either with approved drugs or in clinical trials. For patients with oncogenic aberrations in EGFR, ALK, and ROS1 specific kinase inhibitor therapy has already become standard of care. Genetic aberrations in BRAF, HER2, RET, MET, NTRK, and others are in clinical evaluation. More and more the biological mechanisms underlying the resistance to TKI therapy are understood and enable the successful therapy with next-generation inhibitors, in particular in EGFR-mutated and $A L K$-positive patients. The application of these stratified therapies requires broad molecular testing of tumor biopsies and, in the near future, possibly also blood, at first diagnosis as well as in relapse. In view of the enormous dynamics in the field a particular challenge of this development is the reorganization of the collaboration between highly specialized academic centers, community hospitals, and private practice-based physicians to enable access of all lung cancer patients to these therapeutic options. 


\section{Disclosure Statement}

J.W. has received honoraria for consultancy and lecture fees from AstraZeneca, Bristol-Myers Squibb, Boehringer Ingelheim, MSD, Clovis, Novartis, Pfizer, Roche as well as research support from Bayer, Boehringer Ingelheim, Novartis, Pfizer, and Roche.

S.M. has received honoraria for consultancy and lecture fees from Boehringer Ingelheim, AstraZeneca, Pfizer, and Novartis.

\section{References}

1 Bonomi P, et al.: Comparison of survival and quality of life in advanced non-small-cell lung cancer patients treated with two dose levels of paclitaxel combined with cisplatin versus etoposide with cisplatin: results of an Eastern Cooperative Oncology Group trial. J Clin Oncol 2000;18:623-631.

$\checkmark 2$ Belani CP, et al.: Randomized phase III trial comparing cisplatin-etoposide to carboplatin-paclitaxel in advanced or metastatic non-small cell lung cancer. Ann Oncol 2005; 16:1069-1075

3 Schiller JH, et al.: Comparison of four chemotherapy regimens for advanced non-small-cell lung cancer. $\mathrm{N}$ Engl J Med 2002;346:92-98.

4 Cardenal F, et al.: Randomized phase III study of gemcitabine-cisplatin versus etoposide-cisplatin in the treatment of locally advanced or metastatic non-smallcell lung cancer. J Clin Oncol 1999;17:12-18.

5 Scagliotti GV, et al.: Phase III study comparing cisplatin plus gemcitabine with cisplatin plus pemetrexed in chemotherapy-naive patients with advanced-stage nonsmall-cell lung cancer. J Clin Oncol 2008;26:3543-3551.

6 Reck M, et al.: Phase II study of oral vinorelbine in combination with carboplatin followed by consolidation therapy with oral vinorelbine as single-agent in unresectable localized or metastatic non-small cell lung carcinoma. Lung Cancer 2009;64:319-325.

7 Reck M, et al.: Phase III trial of cisplatin plus gemcitabine with either placebo or bevacizumab as first-line therapy for nonsquamous non-small-cell lung cancer: AVAil. J Clin Oncol 2009;27:1227-1234.

8 Sandler A, et al.: Paclitaxel-carboplatin alone or with bevacizumab for non-small-cell lung cancer. N Engl J Med 2006;355:2542-2550.

$\checkmark 9$ Garon EB, et al.: Ramucirumab plus docetaxel versus placebo plus docetaxel for second-line treatment of stage IV non-small-cell lung cancer after disease progression on platinum-based therapy (REVEL): a multicentre, double-blind, randomised phase 3 trial. Lancet 2014;384:665-673.

10 Rosell R, et al.: Erlotinib versus standard chemotherapy as first-line treatment for European patients with advanced EGFR mutation-positive non-small-cell lung cancer (EURTAC): a multicentre, open-label, randomised phase 3 trial. Lancet Oncol 2012;13:239-246.

11 Maemondo M, et al.: Gefitinib or chemotherapy for non-small-cell lung cancer with mutated EGFR. N Engl J Med 2010;362:2380-2388.

12 Mok TS, et al.: Gefitinib or carboplatin-paclitaxel in pulmonary adenocarcinoma. N Engl J Med 2009;361: 947-957.

13 Mitsudomi T, et al.: Gefitinib versus cisplatin plus docetaxel in patients with non-small-cell lung cancer harbouring mutations of the epidermal growth factor receptor (WJTOG3405): an open label, randomised phase 3 trial. Lancet Oncol 2010;11:121-128.

14 Kawaguchi T, et al.: Randomized phase III trial of erlotinib versus docetaxel as second- or third-line therapy in patients with advanced non-small-cell lung cancer: Docetaxel and Erlotinib Lung Cancer Trial (DELTA). J Clin Oncol 2014;32:1902-1908.
5 Sequist LV, et al.: Phase III study of afatinib or cisplatin plus pemetrexed in patients with metastatic lung adenocarcinoma with EGFR mutations. J Clin Oncol 2013;31:3327-3334.

16 Shaw AT, et al.: Crizotinib versus chemotherapy in advanced ALK-positive lung cancer. N Engl J Med 2013; 368:2385-2394.

17 Shaw AT, Engelman JA: Ceritinib in ALK-rearranged non-small-cell lung cancer. N Engl J Med 2014;370: 2537-2539.

18 Shaw AT, et al.: Alectinib in ALK-positive, crizotinibresistant, non-small-cell lung cancer: a single-group, multicentre, phase 2 trial. Lancet Oncol 2016;17:234242.

19 Barlesi F, et al.: Routine molecular profiling of patients with advanced non-small-cell lung cancer: results of a 1-year nationwide programme of the French Cooperative Thoracic Intergroup (IFCT). Lancet 2016;387: 1415-1426.

20 Kris MG, et al.: Using multiplexed assays of oncogenic drivers in lung cancers to select targeted drugs. JAMA 2014;311:1998-2006.

21 Clinical Lung Cancer Genome project, Network Genomic Medicine: A genomics-based classification of human lung tumors. Sci Transl Med 2013;5:209ra153.

22 Rizvi NA, et al.: Cancer immunology. Mutational landscape determines sensitivity to PD-1 blockade in nonsmall cell lung cancer. Science 2015;348:124-128.

23 Negrini S, et al.: Genomic instability-an evolving hallmark of cancer. Nat Rev Mol Cell Biol 2010;11:220228.

24 Gerlinger M,Swanton C: How Darwinian models inform therapeutic failure initiated by clonal heterogeneity in cancer medicine. Br J Cancer 2010;103:1139-1143.

25 Azam M, et al.: Activation of tyrosine kinases by mutation of the gatekeeper threonine. Nat Struct Mol Biol 2008;15:1109-1118.

26 Katayama R, et al.: Mechanisms of acquired crizotinib resistance in ALK-rearranged lung Cancers. Sci Transl Med 2012;4:120ra17.

27 Cross DA, et al.: AZD9291, an irreversible EGFR TKI, overcomes T790M-mediated resistance to EGFR inhibitors in lung cancer. Cancer Discov 2014;4:1046-1061.

28 Friboulet L, et al.: The ALK inhibitor ceritinib overcomes crizotinib resistance in non-small cell lung cancer. Cancer Discov 2014;4(6):662-673.

29 Ortiz-Cuaran S, et al.: Heterogeneous mechanisms of primary and acquired resistance to third-generation EGFR inhibitors. Clin Cancer Res 2016;22:4837-4847.

30 Bean J, et al.: MET amplification occurs with or without T790M mutations in EGFR mutant lung tumors with acquired resistance to gefitinib or erlotinib. Proc Natl Acad Sci U S A 2007;104:20932-20937.

31 Tricker EM, et al.: Combined EGFR/MEK Inhibition prevents the emergence of resistance in EGFR-mutant lung cancer. Cancer Discov 2015;5(9):960-71.

32 Ercan D, et al.: Reactivation of ERK signaling causes resistance to EGFR kinase inhibitors. Cancer Discov 2012;2:934-947.
33 Lynch TJ, et al.: Activating mutations in the epidermal growth factor receptor underlying responsiveness of non-small-cell lung cancer to gefitinib. N Engl J Med 2004;350:2129-2139.

34 Paez JG, et al.: EGFR mutations in lung cancer: correlation with clinical response to gefitinib therapy. Science 2004;304:1497-1500.

35 Pao W, et al.: EGF receptor gene mutations are common in lung cancers from 'never smokers' and are associated with sensitivity of tumors to gefitinib and erlotinib. Proc Natl Acad Sci U S A 2004;101:13306-13311.

36 Zhou C, et al.: Erlotinib versus chemotherapy as firstline treatment for patients with advanced EGFR mutation-positive non-small-cell lung cancer (OPTIMAL, CTONG-0802): a multicentre, open-label, randomised, phase 3 study. Lancet Oncol 2011;12:735-742.

37 Yang JC, et al.: Afatinib versus cisplatin-based chemotherapy for EGFR mutation-positive lung adenocarcinoma (LUX-Lung 3 and LUX-Lung 6): analysis of overall survival data from two randomised, phase 3 trials. Lancet Oncol 2015;16:141-151.

38 Sequist LV, et al.: Genotypic and histological evolution of lung cancers acquiring resistance to EGFR inhibitors. Sci Transl Med 2011;3:75ra26.

39 Yu HA, et al.: Analysis of tumor specimens at the time of acquired resistance to EGFR-TKI therapy in $155 \mathrm{pa}$ tients with EGFR-mutant lung cancers. Clin Cancer Res 2013;19:2240-2247.

40 Janne PA, et al.: AZD9291 in EGFR inhibitor-resistant non-small-cell lung cancer. N Engl J Med 2015;372: 1689-1699.

41 Tan DS, et al.: First-in-human phase I study of EGF816, a third generation, mutant-selective EGFR tyrosine kinase inhibitor, in advanced non-small cell lung cancer (NSCLC). J Clin Oncol 2015;33:suppl; abstr 8013.

42 Tan DS, et al.: Updated results of a phase 1 study of EGF816, a third-generation, mutant-selective EGFR tyrosine kinase inhibitor (TKI), in advanced non-small cell lung cancer (NSCLC) harboring T790M. J Clin Oncol 2016;34:suppl; abstr 9044.

43 Park K, et al.: BI 1482694 (HM61713), an EGFR mutant-specific inhibitor, in T790M+ NSCLC: Efficacy and safety at the RP2D. J Clin Oncol 2016;34:suppl; abstr 9055 .

44 Chabon JJ, et al.: Circulating tumour DNA profiling reveals heterogeneity of EGFR inhibitor resistance mechanisms in lung cancer patients. Nat Commun 2016;7:11815.

45 Scheffler M, et al.: ROS1 rearrangements in lung adenocarcinoma: prognostic impact, therapeutic options and genetic variability. Oncotarget 2015;6:10577-10585.

46 Thress KS, et al.: Acquired EGFR C797S mutation mediates resistance to AZD9291 in non-small cell lung cancer harboring EGFR T790M. Nat Med 2015;21(6): 560-562.

47 Yu HA, et al.: Acquired resistance of EGFR-mutant lung cancer to a T790M-specific EGFR inhibitor: emergence of a third mutation (C797S) in the EGFR tyrosine kinase domain. JAMA Oncol 2015;1:982-984. 
48 Ramalingam SS, et al.: Osimertinib as first-line treatment for EGFR mutation-positive advanced NSCLC: updated efficacy and safety results from two phase I expansion cohorts. J Thorac Oncol 2016;11:suppl; S152.

49 Takeuchi K, et al.: RET, ROS1 and ALK fusions in lung cancer. Nat Med 2012;18:378-381.

50 Soda $\mathrm{M}$, et al.: Identification of the transforming EML4-ALK fusion gene in non-small-cell lung cancer. Nature 2007;448:561-566.

51 Blackhall FH, et al.: Prevalence and clinical outcomes for patients with ALK-positive resected stage I to III adenocarcinoma: results from the European Thoracic Oncology Platform Lungscape Project. J Clin Oncol 2014;32:2780-2787.

52 Solomon BJ, et al.: First-line crizotinib versus chemotherapy in ALK-positive lung cancer. N Engl J Med 2014;371:2167-2177.

53 Sasaki T, et al.: A novel ALK secondary mutation and EGFR signaling cause resistance to ALK kinase inhibitors. Cancer Res 2011;71:6051-6060.

54 Doebele RC, et al.: Mechanisms of resistance to crizotinib in patients with ALK gene rearranged non-small cell lung cancer. Clin Cancer Res 2012;18:1472-1482.

55 Ou SH, et al.: Alectinib in Crizotinib-Refractory ALKrearranged non-small-cell lung cancer: a phase II global study. J Clin Oncol 2016;34:661-668.

56 Costa DB, et al.: CSF concentrations of the anaplastic lymphoma kinase inhibitor crizotinib. J Clin Oncol 2011;29:e443-e445

57 Costa DB, et al.: Clinical experience with crizotinib in patients with advanced alk-rearranged non-small-cell lung cancer and brain metastases. J Clin Oncol 2015; 33:1881-1888.

58 Kim DW, et al.: Activity and safety of ceritinib in patients with ALK-rearranged non-small-cell lung cancer (ASCEND-1): updated results from the multicentre, open-label, phase 1 trial. Lancet Oncol 2016;17:452463.

59 Gadgeel SM, et al.: Safety and activity of alectinib against systemic disease and brain metastases in patients with crizotinib-resistant ALK-rearranged nonsmall-cell lung cancer (AF-002JG): results from the dose-finding portion of a phase $1 / 2$ study. Lancet Oncol 2014;15:1119-1128.

60 Solomon BJ, et al.: Intracranial efficacy of crizotinib versus chemotherapy in patients with advanced ALKpositive non-small-cell lung cancer: results from PROFILE 1014. J Clin Oncol 2016;34:2858-2865.

61 Bergethon K, et al.: ROS1 rearrangements define a unique molecular class of lung cancers. J Clin Oncol 2012;30:863-870.

62 Davies KD, et al.: Identifying and targeting ROS1 gene fusions in non-small cell lung cancer. Clin Cancer Res 2012;18:4570-4579.

63 Mazieres J, et al.: Crizotinib therapy for advanced lung adenocarcinoma and a ROS1 rearrangement: results from the EUROS1 cohort. J Clin Oncol 2015;33:992999.

64 Davare MA, et al.: Structural insight into selectivity and resistance profiles of ROS1 tyrosine kinase inhibitors. Proc Natl Acad Sci U S A 2015;112:E5381-5390.

65 Drilon A, et al.: A novel crizotinib-resistant solventfront mutation responsive to cabozantinib therapy in a patient with ROS1-rearranged lung cancer. Clin Cancer Res 2016;22:2351-2358.

66 Facchinetti F, et al.: Crizotinib-resistant ROS1 mutations reveal a predictive kinase inhibitor sensitivity model for ROS1- and ALK-rearranged lung cancers. Clin Cancer Res 2016; DOI: 10.1158/1078-0432.CRR16-0917.

67 Katayama R, et al.: Cabozantinib overcomes crizotinib resistance in ROS1 fusion-positive cancer. Clin Cancer Res 2015;21:166-174
68 Chong CR, et al.: Identification of existing drugs that effectively target NTRK1- and ROS1-rearrangements in lung cancer. Clin Cancer Res 2016; DOI: 10.1158/ 1078-0432.CRR-15-1601.

69 Davies KD, et al.: Resistance to ROS1 inhibition mediated by EGFR pathway activation in non-small cell lung cancer. PLoS One 2013;8:e82236.

70 Cargnelutti M, et al.: Activation of RAS family members confers resistance to ROS1 targeting drugs. Oncotarget 2015;6:5182-5194

71 Solomon BJ, et al.: Safety and efficacy of lorlatinib (PF06463922) from the dose-escalation component of a study in patients with advanced ALK+ and ROS1+ non-small cell lung cancer (NSCLC). J Clin Oncol 2016;34:suppl; abstr 9009.

72 Schildhaus HU, et al.: MET amplification status in therapy-naive adeno- and squamous cell carcinomas of the lung. Clin Cancer Res 2015;21:907-915.

73 Tong JH, et al.: MET amplification and exon 14 splice site mutation define unique molecular subgroups of non-small cell lung carcinoma with poor prognosis. Clin Cancer Res 2016;22:3048-3056.

74 Lennerz JK, et al.: MET amplification identifies a small and aggressive subgroup of esophagogastric adenocarcinoma with evidence of responsiveness to crizotinib. J Clin Oncol 2011;29:4803-4810.

75 Dulak AM, et al.: Gastrointestinal adenocarcinomas of the esophagus, stomach, and colon exhibit distinct patterns of genome instability and oncogenesis. Cancer Res 2012;72:4383-4393.

76 Dziadziuszko R, et al.: Correlation between MET gene copy number by silver in situ hybridization and protein expression by immunohistochemistry in nonsmall cell lung cancer. J Thorac Oncol 2012;7:340-347.

77 Awad MM, et al.: MET Exon 14 Mutations in NonSmall-Cell Lung Cancer Are Associated With Advanced Age and Stage-Dependent MET genomic amplification and c-Met overexpression. J Clin Oncol 2016;34:721-730.

78 Cappuzzo F, et al.: Increased MET gene copy number negatively affects survival of surgically resected nonsmall-cell lung cancer patients. J Clin Oncol 2009;27: 1667-1674.

79 Spigel DR, et al.: Randomized phase II trial of onartuzumab in combination with erlotinib in patients with advanced non-small-cell lung cancer. J Clin Oncol 2013;31:4105-4114.

80 Schwab R, et al.: Major partial response to crizotinib, a dual MET/ALK inhibitor, in a squamous cell lung (SCC) carcinoma patient with de novo c-MET amplification in the absence of ALK rearrangement. Lung Cancer 2014;83:109-111.

81 Schuler $\mathrm{MH}$, et al.: Phase (Ph) I study of the safety and efficacy of the cMET inhibitor capmatinib (INC280) in patients (pts) with advanced cMET+ non-small cell lung cancer (NSCLC). J Clin Oncol 2016;34:suppl; abstr 9067.

82 Paik PK, et al.: Response to MET inhibitors in patients with stage IV lung adenocarcinomas harboring MET mutations causing exon 14 skipping. Cancer Discov 2015;5:842-849.

83 Camidge DR, et al.: Efficacy and safety of crizotinib in patients with advanced c-MET-amplified non-small cell lung cancer (NSCLC). J Clin Oncol 2014;32:suppl; abstr 8001.

84 Cardarella S, et al.: Clinical, pathologic, and biologic features associated with BRAF mutations in non-small cell lung cancer. Clin Cancer Res 2013;19:4532-4540.

85 Kinno T, et al.: Clinicopathological features of nonsmall cell lung carcinomas with BRAF mutations. Ann Oncol 2014;25:138-142.

86 Paik PK, et al.: Clinical characteristics of patients with lung adenocarcinomas harboring BRAF mutations. J Clin Oncol 2011;29:2046-2051.
McArthur GA, et al.: Safety and efficacy of vemurafenib in $\mathrm{BRAF}(\mathrm{V} 600 \mathrm{E})$ and $\mathrm{BRAF}(\mathrm{V} 600 \mathrm{~K})$ mutation-positive melanoma (BRIM-3): extended follow-up of a phase 3, randomised, open-label study. Lancet Oncol 2014;15:323-332

88 Long GV, et al.: Dabrafenib and trametinib versus dabrafenib and placebo for Val600 BRAF-mutant melanoma: a multicentre, double-blind, phase 3 randomised controlled trial. Lancet 2015;386:444-451.

89 Planchard D, et al.: Dabrafenib plus trametinib in patients with previously treated BRAF(V600E)-mutant metastatic non-small cell lung cancer: an open-label, multicentre phase 2 trial. Lancet Oncol 2016;17:984993.

90 Hyman DM, et al.: Vemurafenib in multiple nonmelanoma cancers with BRAF V600 mutations. N Engl J Med 2015;373:726-736.

91 Saunders LR, et al.: A DLL3-targeted antibody-drug conjugate eradicates high-grade pulmonary neuroendocrine tumor-initiating cells in vivo. Sci Transl Med 2015;7:302ral36.

92 Rudin C, et al.: Safety and efficacy of single-agent rovalpituzumab teserine (SC16LD6.5), a delta-like protein 3 (DLL3)-targeted antibody-drug conjugate (ADC) in recurrent or refractory small cell lung cancer (SCLC). J Clin Oncol 2016;34:suppl; abstr LBA8505.

93 Weiss J, et al.: Frequent and focal FGFR1 amplification associates with therapeutically tractable FGFR1 dependency in squamous cell lung cancer. Sci Transl Med 2010;2:62ra93.

94 Zhang H, et al.: Enhanced FGFR signalling predisposes pancreatic cancer to the effect of a potent FGFR inhibitor in preclinical models. Br J Cancer 2014;110:320329.

95 Guagnano V, et al.: FGFR genetic alterations predict for sensitivity to NVP-BGJ398, a selective pan-FGFR inhibitor. Cancer Discov 2012;2:1118-1133.

96 Soria JC, et al.: Phase I/IIa study evaluating the safety, efficacy, pharmacokinetics, and pharmacodynamics of lucitanib in advanced solid tumors. Ann Oncol 2014; 25:2244-2251.

97 Lim SH, et al.: Efficacy and safety of dovitinib in pretreated patients with advanced squamous non-small cell lung cancer with FGFR1 amplification: A singlearm, phase 2 study. Cancer 2016;122:3024-3031.

98 Nogova L, et al.: Targeting FGFR1-amplified lung squamous cell carcinoma with the selective pan-FGFR inhibitor BGJ398. J Clin Oncol 2014;32:suppl; abstr 8034.

99 Farago AF, et al.: Durable clinical response to entrectinib in NTRK1-rearranged non-small cell lung cancer. J Thorac Oncol 2015;10:1670-1674.

100 Vaishnavi A, et al.: Oncogenic and drug-sensitive NTRK1 rearrangements in lung cancer. Nat Med 2013; 19:1469-1472.

101 Vaishnavi, A., A.T. Le, and R.C. Doebele, TRKing down an old oncogene in a new era of targeted therapy. Cancer Discov 2015. 5(1):25-34.

102 Ardini E, et al.: Entrectinib, a pan-TRK, ROS1, and ALK inhibitor with activity in multiple molecularly defined cancer indications. Mol Cancer Ther 2016;15: 628-639.

103 Drilon A, et al.: A phase II basket study of the oral TRK inhibitor LOXO-101 in adult subjects with NTRK fusion-positive tumors. J Clin Oncol 2016;34:suppl; abstr TPS2599.

104 de Braud F, et al.: Alka-372-001: first-in-human, phase I study of entrectinib - an oral pan-trk, ROS1, and ALK inhibitor - in patients with advanced solid tumors with relevant molecular alterations. J Clin Oncol 2015;33:suppl; abstr 2517.

105 Mazieres J, et al.: Lung cancer patients with HER2 mutations treated with chemotherapy and HER2-targeted drugs: results from the European EUHER2 cohort. Ann Oncol 2016;27:281-286. 
106 Mazieres J, et al.: Lung cancer that harbors an HER2 mutation: epidemiologic characteristics and therapeutic perspectives. J Clin Oncol 2013;31:1997-2003.

107 Drilon A, et al.: Response to cabozantinib in patients with RET fusion-positive lung adenocarcinomas. Cancer Discov 2013;3:630-635.

108 Konig K, et al.: Implementation of amplicon paralle sequencing leads to improvement of diagnosis and therapy of lung cancer patients. J Thorac Oncol 2015; 10:1049-1057.
109 Drilon A, et al.: Broad, hybrid capture-based next-generation sequencing identifies actionable genomic alterations in lung adenocarcinomas otherwise negative for such alterations by other genomic testing approaches. Clin Cancer Res 2015;21:3631-3639.

110 Yanagita M, et al.: A prospective evaluation of circulating tumor cells and cell-free DNA in EGFR mutant non-small cell lung cancer patients treated with erlotinib on a phase II trial. Clin Cancer Res 2016; DOI: 10.1158/1078-0432.CRR-16-0909.
111 Watanabe M, et al.: Ultra-sensitive detection of the pretreatment EGFR T790M Mutation in Non-Small Cell Lung Cancer Patients with an EGFR-activating mutation using droplet digital PCR. Clin Cancer Res 2015,21:3552-3560.

112 Swanton C, et al.: Consensus on precision medicine for metastatic cancers: a report from the MAP conference. Ann Oncol 2016,27:1443-1448. 